\title{
Selective induction of human gut-associated acetogenic/ butyrogenic microbiota based on specific microbial colonization of indigestible starch granules
}

\author{
Yusuke Nagara $\mathbb{D}^{1 凶}{ }^{凶}$, Daichi Fujii $\mathbb{D}^{1}{ }^{1}$, Toshihiko Takada ${ }^{1}$, Mikiko Sato-Yamazaki ${ }^{1}$, Toru Odani $\mathbb{I D}^{1}$ and Kenji Oishi $^{1}$ \\ (c) The Author(s) 2022
}

\begin{abstract}
Prediction of individualized responses is one of biggest challenges in dietary intervention to modulate human gut microbiota. Bacterial interspecies competition for dietary factors should underlie the inter-subject heterogeneity of microbial responses. Microscale localization of bacterial species around intestinal food structures could provide direct evidence for understanding this, however, little information is currently available. Here we analyzed human fecal sections and found multiple types of bacterial colonization of food structures. The most eminent one was dense and frequent colonization of starch granules by Bifidobacterium adolescentis. After intake of raw potato starch (pSt), B. adolescentis dramatically increased in every carrier of the species, accompanied by an increase in bifidobacterial metabolite acetate. In the other subjects, Eubacterium rectale and its metabolite butyrate increased, but it was suppressed in B. adolescentis carriers. A correlation analysis indicated the contribution of these species to respective metabolites. In vitro analyses of isolates of major gut bacterial species confirmed that these species are major colonizers of pSt and that $B$. adolescentis can colonize pSt even in the presence of the known starch granule-degrading bacterium Ruminococcus bromii. Collectively, we propose that specific binding of $B$. adolescentis or $E$. rectale to pSt selectively induces acetogenic or butyrogenic response of gut microbiota, where the former determines the response of the latter.
\end{abstract}

The ISME Journal (2022) 16:1502-1511; https://doi.org/10.1038/s41396-022-01196-w

\section{INTRODUCTION}

The intestinal microbiota contributes to many aspects of host health and diseases via bioactive metabolites including shortchain fatty acids (SCFAs). Diet is a rapid and powerful tool for modulating human microbiota and their metabolism [1-3]. A specific set of gut bacteria expands upon taking up each food, including indigestible polysaccharides, in some cases after degradation by host or microbial enzymes [4-7]. Direct utilization of each nutrient in the intestine by specific human gut-associated bacteria has been demonstrated using isolated strains and recently predicted from genomic data. However, it is still generally impossible to predict individualized responses to dietary intervention, and this will be the biggest limitation in the application of dietary intervention. To overcome this, it is necessary to thoroughly understand the nutritional ecology of gut bacteria, including specific competitions for each nutrient, and key behaviors that make bacteria more competitive in situ and enable them to take up nutrients before competitors consume them. A recent study showed a conceptual example of interspecies competition for a nutrient by using gnotobiotic mice [8], however, competitions and key behaviors are largely unidentified in vivo or in situ by analyzing normal human intestinal microbiota. To address this, we focused on the distribution of bacteria around food structures in human feces. We show specific bacterial colonization of a specific type of starch in human fecal sections, and also show the impact of the major colonizing species on the response of microbiota to a nutrient that is competed for by multiple gut bacterial species. We found that efficient utilization of starch granules was nearly monopolized by the major colonizing species Bifidobacterium adolescentis, although another colonizing species, Eubacterium rectale, also can bind and respond to the granules. The major gut fermentation product in each subject was determined by which of the two species responded and especially by the presence of $B$. adolescentis.

\section{MATERIALS AND METHODS}

\section{Human studies and ethical approval}

All human studies were conducted in accordance with the Declaration of Helsinki. The studies were scientifically and ethically reviewed and were approved by the Yakult Central Institute. Written informed consent was obtained from the participants after explanation of the study (aims, measurements, possible risks and consequences) before enrollment.

Experiment I. Five healthy male adults (age, 28-53 years) who had not taken antibiotics for at least one week prior to the day of the first sampling participated. Fecal specimens were collected twice with a 2-week interval. Subjects were asked to avoid overeating or overdrinking during the period. Contents of meals for 2 days before sampling were surveyed.

Experiment II. Ten healthy male adults (age, 29-56 years) were recruited. This experiment was designed according to a previous study [9]. After 10 days of pre-intake period, the participants were asked to consume raw

${ }^{1}$ Microbiological Research Department, Yakult Central Institute, Kunitachi, Tokyo, Japan. ${ }^{\varpi_{e}}$ email: yusuke-nagara@yakult.co.jp 
potato starch (hereafter referred as pSt, unmodified potato starch, Bob's Red Mill, Milwaukie, OR, USA) as follows: on the first day, one serving of 12 $\mathrm{g}$; on the second day, one serving of $24 \mathrm{~g}$; and then $48 \mathrm{~g} /$ day divided into equal 2 servings for 12 days. Fecal specimens were collected four times between the 3rd and 10th days of the pre-intake period and four times between the 4th and 14th days of the pSt intake period, with at least oneday interval between sampling in most cases. Intake of banana, any raw tubers, raw starch powder (other than that provided by the researcher), or large amounts of chilled potato salad was prohibited throughout the experiment to avoid introduction of large amounts of granular resistant starch to the intestine.

Experiment III. Ten healthy male adults (age, 28-54 years) were recruited. After 9 days of the pre-intake period, they were asked to take $40 \mathrm{~g} /$ day of raw Japanese yam (mix of Dioscorea japonica and D. polystachya, contains approx. $14 \%$ [w/w] of granular starch, Maruko Foods, Saitama, Japan) at lunch time for 4 days, followed by 8 days of the post-intake period (starchy foods are rarely consumed raw, but Japanese yam is an exceptionally common raw starchy food in Japan; after examining meal logs, it was the only candidate source of $B$. adolescentis-colonized starch granules in Experiment I). Six fecal specimens were collected in total from each subject (two for each period). Intake of banana and additional raw Japanese yam (other than that provided by the researcher) was prohibited during all periods of this experiment. Two subjects ( $F$ and $G$ ) were recruited again after the experiment, another informed consent was obtained, and fecal material was collected once for isolation of dominant bacteria. For this sampling, food intake was not restricted.

\section{Analysis of fecal sections}

Fecal sections were prepared as described by Swidsinski et al. [10]. A fraction of fresh feces was sampled by subjects by puncturing it with a plastic straw (diameter $6 \mathrm{~mm}$; length of the fecal specimen $\sim 1-2 \mathrm{~cm}$ ). The sample was kept cold using a cold pack (for up to $4 \mathrm{~h}$ ) and fixed with methacarn fixative [11] for 2-4h. The fixed sample was embedded first into $1 \%$ agarose and then into paraffin. Sections of $4 \mu \mathrm{m}$ were obtained for the following analyses.

FISH staining was performed as previously described [12]. Briefly, sections were deparaffinized and incubated with a hybridization buffer containing $4.5 \mathrm{ng} / \mu \mathrm{l}$ of probes (Supplementary Table 1). To stain mucus (glycoproteins), sections were incubated with alcian blue at $\mathrm{pH} 2.5$ and then with periodic acid-Schiff (PAS). Alcian blue was purchased from Merck Millipore (Burlington, MA, USA) and reagents for PAS staining from Muto Pure Chemicals (Tokyo, Japan). For iodine staining, sections were incubated with Lugol's iodine solution (Muto Pure Chemicals) for $30 \mathrm{~s}$ and then washed with water for about $10 \mathrm{~s}$.

Specimens were mounted using Vectashield with DAPI (Vector Laboratories, Burlingame, CA, USA) for fluorescent microscopy, or using Entellan New (Merck Millipore) for observation of iodine staining without FISH and of alcian blue-PAS staining. For double staining (FISH and iodine), specimens were first stained by FISH and then by iodine, and mounted using Vectashield with DAPI. Before mounting, more than ten independent fields of view containing one or more deep-purple starch granules were randomly selected for each slide and memorized by the microscope operating software MetaMorph (Molecular Devices, San Jose, (A, USA); immediately after mounting, observation of stained starch and image acquisition were performed using memorized positions (iodine staining fades soon after mounting, presumably because of redox reaction between mounting media and iodine; fading of starch granules is slower than that of soluble starch). Fluorescence was observed using a DM6000B microscope system (Leica Microsystems, Wetzlar, Germany). Monochrome images were acquired for each fluorescent dye and pseudocolors were assigned to make overlay images. Bright-field images for starch granule measurements were obtained under a BZX700 microscope (Keyence, Osaka, Japan).

To confirm the specificity of starch staining, serial sections were incubated with $0.5 \%$ porcine amylase (A3176, Sigma-Aldrich, St. Louis, MO, USA) in PBS at $37^{\circ} \mathrm{C}$ for $2 \mathrm{~h}$ and then stained with Lugol's solution.

A macro program that selects starch granules but not solubilized starch was designed in Image-Pro Plus 6 (Media Cybernetics, Rockville, MD, USA). Representative images containing average amounts of starch granules in each specimen were analyzed by this macro to calculate the percentage of starch granule area in the field. Area outside of fecal material and area occupied by air bubbles were omitted from the calculation. Area of excreted starch granules (\%) was calculated as [area of starch granules during the intake period (\%)] - [that during the pre-intake period (\%)].

\section{Microbial profiling}

Microbial profiling based on $16 \mathrm{~S}$ rRNA gene sequences was performed as previously described [13]. DNA was extracted from $20 \mathrm{mg}$ of feces by bead-phenol method [14] and dissolved in $1 \mathrm{ml}$ of TE buffer. The V1-V2 region of the $16 \mathrm{~S}$ rRNA gene was amplified by PCR using DNA $(1 \mu \mathrm{l})$ as a template, TB Green Premix Ex Taq II (Tli RNaseH Plus) (Takara Bio, Shiga, Japan), and primers (27Fmod2-MiSeq and 338R-MiSeq, Supplementary Table 1). To prevent erroneous amplification, the reaction was stopped when the TB Green signal was close to saturation. The products were purified using Agencourt AMPure XP beads (Beckman Coulter, Brea, CA, USA) and all samples were pooled to make a library. The library was sequenced using a MiSeq Reagent Kit v2 (500 cycles) and a MiSeq instrument (Illumina, San Diego, CA, USA). Raw sequence data were deposited in DDBJ DRA under accession number PRJDB11235.

\section{Measurement of SCFAs}

SCFAs were measured as previously described [6]. Fecal samples were suspended in a 9-fold volume of PBS, and an aliquot of the suspension was mixed with one-ninth volume of $10 \%$ perchloric acid. The mixture was tightly sealed and kept at $4{ }^{\circ} \mathrm{C}$ until analysis. On the day of analysis, the samples were filtered through Centricut Ultramini filters (\#W-MO-045, Kurabo, Osaka, Japan) and analyzed by HPLC with pure SCFAs (lithium lactate, sodium acetate, sodium propionate and sodium butyrate) at known concentrations as standards.

\section{Measurement of total fecal bacterial count}

Fecal samples were washed with PBS, resuspended in PBS containing $0.1 \%$ Tween-80, sonicated and centrifuged at $100 \times g$ for $1 \mathrm{~min}$ to remove large debris. Aliquots of the supernatants were mounted using Vectashield with DAPI, and fluorescence of DAPI was imaged for ten independent fields of view using Leica DM6000B microscope. The cells in these images were counted using Image-Pro Plus 6.

\section{Isolation of major gut bacterial species}

Fresh fecal samples or those stored at $-80^{\circ} \mathrm{C}$ were used. A small fraction of each sample was diluted and plated onto TOS propionate agar (Yakult Pharmaceutical Industry, Tokyo, Japan) supplemented with $50 \mu \mathrm{g} / \mathrm{ml}$ mupirocin, or BL agar (Nissui, Tokyo, Japan) supplemented with $5 \%$ defibrinated horse blood. Plates were incubated for $1-2$ days at $37^{\circ} \mathrm{C}$ in an anaerobic glove box. Colonies were sorted into types by their morphology. For each type, several single colonies were isolated and subjected to taxonomic identification by $16 \mathrm{~S}$ rRNA gene sequencing (Supplementary Table 1). Sequences were analyzed by NCBI BLAST against the bacterial $16 \mathrm{~S}$ rRNA gene database; $97 \%$ identity was used as a threshold to assign strains to species. Bifidobacterial isolates were additionally typed by RAPD (analysis of random amplified polymorphic DNA) using three primers (Supplementary Table 1). An isolate was chosen per RAPD type as a representative and was evaluated in vitro. For species other than bifidobacteria, a single strain was randomly chosen per species per subject and evaluated.

\section{In vitro evaluation of isolates}

Binding to starch granules was analyzed as follows. Bacterial strains were grown anaerobically on modified GAM agar (Nissui, contains $0.5 \%$ soluble starch as the major carbon source). Approximately $1 \mu$ lof a colony was taken with an inoculating loop and suspended in $150 \mu \mathrm{l}$ of PBS; then, $12 \mu \mathrm{l}$ of this suspension, $80 \mu \mathrm{l}$ of pSt suspension (1\% w/v in PBS), and $88 \mu \mathrm{l}$ of PBS were mixed by vortexing, and formation of bacteria-starch aggregates was examined. If aggregation occurred and the flocks fell immediately to the bottom of the tube making the supernatant transparent, the strain was judged positive. If no aggregation was observed, the strain was judged negative. In negative cases and in the negative control (no bacteria), visible precipitation of starch requires more than $\sim 15 \mathrm{~s}$. Strains that showed selfaggregation (i.e., could not be dispersed or aggregated without starch added) were categorized as undeterminable.

Starch-degrading ability was screened as follows. Strains were grown anaerobically on modified GAM agar, the culture plates were flooded with $1 / 2$ diluted Lugol's solution for about $30 \mathrm{~s}$ and then the solution was removed. Starch-degrading ability was judged from a clear zone around the colonies. When the diameter of this zone was $<\sim 1 \mathrm{~mm}$ or staining around the colony was reduced but was clearly visible, the strain was judged as weakly positive.

Utilization of pSt was analyzed as follows. The powder of GAM Semisolid without Dextrose (Nissui; does not contain starch) was thoroughly 
dissolved in water, agar was removed by precipitation, and the solution was autoclaved at $115^{\circ} \mathrm{C}$ for $15 \mathrm{~min}$. Broths containing different carbon sources were prepared by adding $1 \% \mathrm{w} / \mathrm{v}$ glucose, fructose, or $\mathrm{pSt}$ before autoclaving. All broths were introduced into an anaerobic chamber at least 1 or 2 days before use. A separate broth was prepared by adding $1 \% \mathrm{w} / \mathrm{v}$ pSt into the autoclaved broth without any additional carbon source, in an anaerobic chamber just before starting culture; starch used in this broth had been UV-irradiated in advance for at least $16 \mathrm{~h}$. Bacterial strains were grown anaerobically on modified GAM agar. Each colony $(\sim 1 \mu \mathrm{l})$ was suspended in $150 \mu \mathrm{l}$ PBS, and $5 \mu \mathrm{l}$ was inoculated into each broth and incubated at $37{ }^{\circ} \mathrm{C}$ in the anaerobic chamber for 3 days, and then $\mathrm{pH}$ of the culture was measured.

\section{In vitro competition assay}

Bifidobacterium adolescentis G202, Ruminococcus bromii $\mathrm{YIT} 6078^{\top}$, and $R$. bromii ATCC 51896 were grown anaerobically on modified GAM broth at $37^{\circ} \mathrm{C}$. Fully grown cultures of either of two $R$. bromii strains and G202 were inoculated into GAM broth supplemented with $0.5 \%$ pSt (pSt-GAM) at an approximate 2:1 ratio and incubated for $24 \mathrm{~h}$. Alternatively, $R$. bromii YIT $6078^{\top}$ was grown on pSt-GAM broth for one day, then G202 was inoculated, and the resultant culture was incubated for $48 \mathrm{~h}$.

Culture was sampled at the indicated time points. "Whole" samples were obtained by centrifuging the culture at $13,000 \mathrm{rpm}$ for $10 \mathrm{~s}$. "pStassociated" samples were obtained as follows: the culture was centrifuged at $100 \times g$ for $10 \mathrm{~s}$, the supernatant was removed, PBS was added to the pellet and resuspended, the sample was centrifuged as above and the supernatant was discarded. The pellets were heated at $95^{\circ} \mathrm{C}$ for $15 \mathrm{~min}$ in TE, centrifuged at $13,000 \mathrm{rpm}$ for $1 \mathrm{~min}$, and the supernatants were used as PCR templates. PCR was performed using Premix Ex Taq Hot Start Version (Takara) and equal amounts of three primers: 27Fmod2-notag, g-Bifid-R, and Clept866mR (Supplementary Table 1). We confirmed that when the two species were mixed in arbitrary ratios after measuring $O D$, the corresponding PCR bands were detected in the expected ratio.

\section{Data analysis and statistics}

Output fastq files were analyzed in QIIME2 software (version 2018.11) and its plugins [15]. Noise and chimeras were removed and the sequences were trimmed using the DADA2 plugin [16] with the following settings: denoise-paired --p-trim-left-f 20 --p-trim-left-r 17 --p-trunc-len-f 220 --ptrunc-len-r 200. For Experiment II, a total of $3.1 \mathrm{M}$ reads were obtained (17K-76 K per sample, average $39 \mathrm{~K}$ ), and for Experiment III, a total of $0.8 \mathrm{M}$ reads were obtained $(6 \mathrm{~K}-36 \mathrm{~K}$ per sample, average $14 \mathrm{~K})$. Amplicon sequence variants (ASVs) were classified in family level using the featureclassifier plugin [17] (classify-sklearn method). The classifier was trained on the SILVA138 database using the feature-classifier plugin [17] (fit-classifiernaive-bayes method). Most relevant species of ASVs were identified using vsearch [18] and the NCBI database 16S RefSeq (BioProject IDs: 33175 and 33317, downloaded on 11 January 2019). ASVs were aligned using the $R$ function AlignSegs in the DECIPHER package [19]. A distance matrix was built from the aligned data using the DECIPHER function DistanceMatrix; this matrix was used to identify clusters using the DECIPHER function IdClusters with the "UPGMA" method and a cutoff value of 0.03 to make 97\% OTUs. OTU representative ASVs were chosen by the highest abundance in the OTU and each OTU was labeled by vsearch result of the representative ASV. Because of high $16 \mathrm{~S}$ rRNA similarity (98.4\%), OTUs classified as either $B$. adolescentis or $B$. faecale (or a retracted species $B$. stercoris) were denoted as $B$. adolescentis in this study.

OTUs whose abundance increased in response to pSt intake were identified using DESeq2 [20] with a design of " subject + period", where "period" means the pre-intake or pSt intake period. Raw read counts were normalized by GMPR [21]. The log2 fold changes (LFC) were shrunk using DESeq2 function IfcShrink (type $=$ "apeglm") [22]. Since we used a threshold of 1 for the absolute LFC, the $s$ value represents the "false sign or small" (FSOS) rate, where "small" denotes an absolute LFC less than 1. In identification of significantly increased OTUs, we analyzed the data from $B$. adolescentis carriers (subjects $\mathrm{K}, \mathrm{M}, \mathrm{O}, \mathrm{Q}, \mathrm{R}, \mathrm{T}$ ) and non-carriers (subjects $\mathrm{L}$, $\mathrm{N}, \mathrm{P}, \mathrm{S}$ ) separately.

To determine whether there was a significant change in SCFA concentration from the pre-intake period to the pSt intake period, we performed a Wilcoxon signed-rank test using the R-package exactRankTests [23] function wilcox.exact. We tested the data from $B$. adolescentis carriers and non-carriers separately and adjusted the $p$ value using the Benjamini-Hochberg correction [24] for multiple comparisons.
Spearman's rank correlation coefficient (Spearman's $\rho$ ) and $p$ value between the abundance of OTUs and SCFA concentrations were calculated using the R-package Hmisc [25] function rcorr. The GMPR-normalized OTU count data and SCFA concentration data from 80 samples were divided into 20 groups by subject and period (pre-intake or pSt intake), and averaged within each group. Spearman's $\rho$ values were also calculated between average OTU counts. A network plot was generated using the R-package visNetwork [26].

Excretion of starch granules (\% area) was compared between $B$. adolescentis carriers and non-carriers using two-sided Welch's $t$ test.

\section{RESULTS}

To explore the nutrient-harvesting strategies of human gut bacteria in vivo, we examined the microscale localization of bacteria around intestinal nutrients (Experiment I). This method has been employed in a murine study and proven useful for elucidating how specific bacteria acquire nutrients in the intestine [12]. Stool samples were collected from five subjects who were taking habitual diet, and fecal paraffin sections were analyzed by fluorescence in situ hybridization (FISH) with probes specific for major bacterial taxa. Plant-like structures and bacteria were found in every specimen. Particle density differed substantially among subjects (Supplementary Fig. 1a and b). Several types of bacterial localization were found (Fig. 1 and Supplementary Fig. 1c). Food structures colonized by bacteria in some subjects were often not found in the others, reflecting diversity in diet. In every case, local accumulation of bacteria of the same taxa or morphology was evident, which was distinct from diffuse distribution (Supplementary Fig. 1d). The most eminent feature was colonization of bifidobacteria on undigested starch granules, which have been known as a type of resistant starch (resistant starch type 2: RS2) (Fig. 1a and Supplementary Fig. 1e). Granule surfaces were densely covered by bifidobacteria, which were identified as a starch-utilizing bacterium, B. adolescentis [27], by using a species-specific probe (Fig. 1a). We also found other features, for example, an unspecified clade of Lachnospiraceae localized along a plant exodermis-like cell layer (Fig. 1b), and some bifidobacteria and Lachnospiraceae bacteria enriched near the mucus layer (Fig. 1c).

Bacterial colonization of a nutrient suggests that the nutrient is utilized by or serves as an important habitat for the bacteria. Dense colonization of starch granules by $B$. adolescentis led us to speculate that the species is a key degrader, which breaks down a large fraction of starch granules, possibly providing oligosaccharides, or a key consumer, which takes up and metabolizes a large fraction of starch granules, and affects the overall response of the microbiota to the nutrient. To test this hypothesis, we next checked the response of $B$. adolescentis and whole-gut microbiota to intake of starch granules in ten healthy adults and evaluated how the presence or absence of the species affects the response of the microbiota (Experiment II; Fig. 2a). Commonly consumed purified potato starch (pSt) was used as a source of starch granules, as in a previous study [28]. Intake of $48 \mathrm{~g} /$ day pSt for 2 weeks did not considerably change total bacterial count (Supplementary Fig. 2), but it resulted in a massive increase in Bifidobacteriaceae including $B$. adolescentis in all $B$. adolescentis carriers (Subjects K, M, O, Q, R, T; Fig. 2b-d). Relative abundance of this species increased from $0.1 \%-28.9 \%$ to $32.0 \%-77.6 \%$ (Fig. 2c). Colonization of the species on starch granules was reproducible in every B. adolescentis carrier (Fig. 3a, Supplementary Fig. 3). In subjects without $B$. adolescentis ( $B$. adolescentis non-carriers; Subjects $L, N, P, S)$, the abundance of another known starchutilizing species, Eubacterium rectale [29], increased substantially, from $2.7-15.2$ to $12.6-45.9 \%$ (Fig. 2c). Yet another major known starch-utilizing species, $R$. bromii [30], also showed some increase in two subjects (Subjects P, S; Fig. 2c). A sporadic increase was also found in Ruminococcus torques (Supplementary Fig. 4). 
a
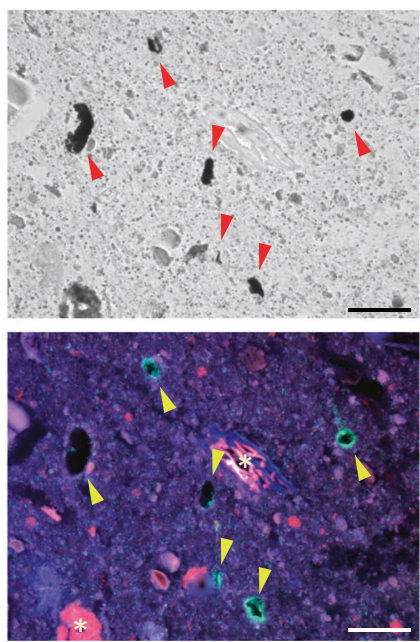

b
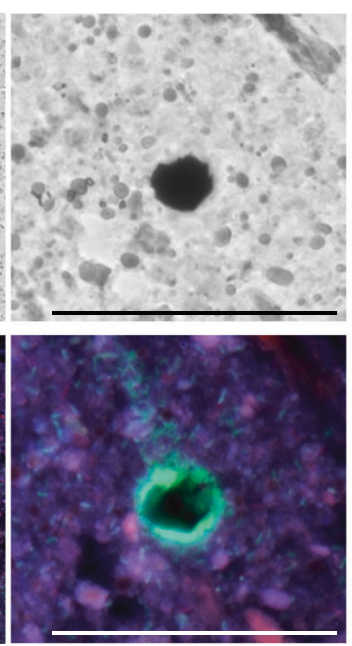

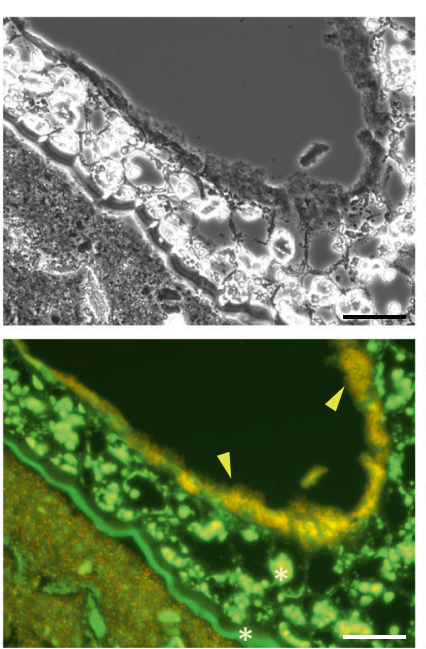

C
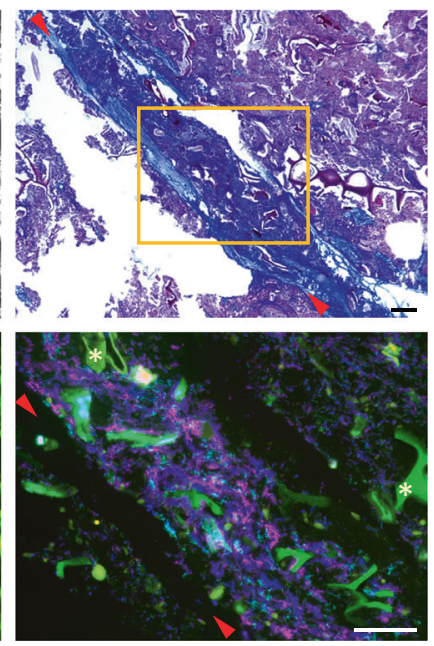

Fig. 1 Microscopic survey of bacterial localization around materials in human feces. a Colonization of $B$. adolescentis on starch granules (arrowheads). Upper: iodine staining; Lower: FISH (Green: B. adolescentis, red: B. catenulatum group, blue: genus Bifidobacterium); B. adolescentis appears cyan because its green signal is merged with the blue signal from genus Bifidobacterium. Images on the right are high-power images of those on the left. b Colonization of Lachnospiraceae on plant tissue (arrowheads). Upper: phase contrast. Lower: FISH (Green: Lachnospiraceae, red: total bacteria). Lachnospiraceae appears yellow because its green signal is merged with the red signal from total bacteria. c Localization of Bifidobacterium and Lachnospiraceae near mucus layer (between arrowheads). Upper: Alcian blue PAS staining. Lower: Fluorescent image of a serial section corresponding to the rectangle in upper image (Green: genus Bifidobacterium, red: Lachnospiraceae, blue: total bacteria). Bars $=50 \mu \mathrm{m} .{ }^{*}$, Autofluorescence from plant-like structures.

a

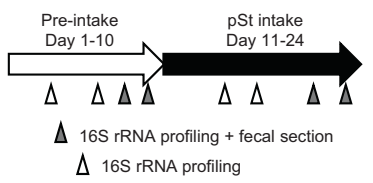

d

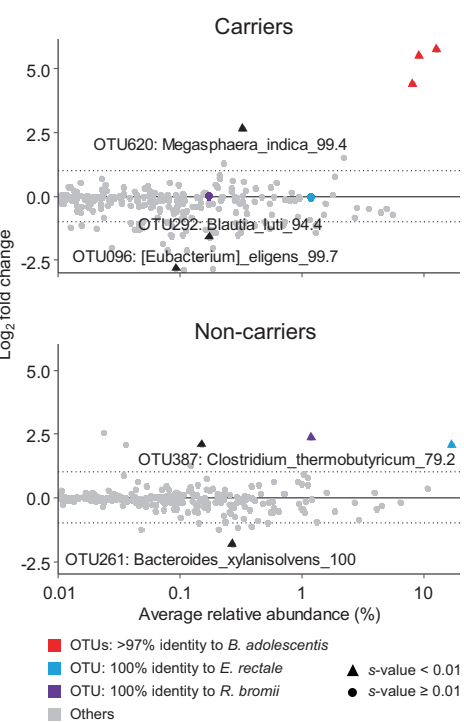

b

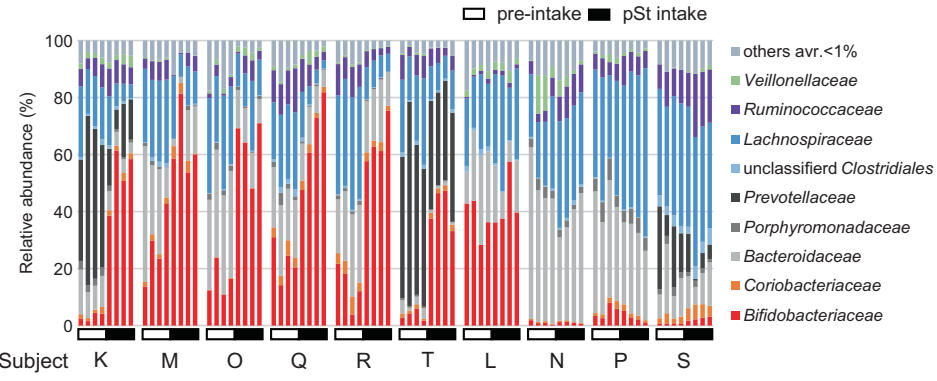

C

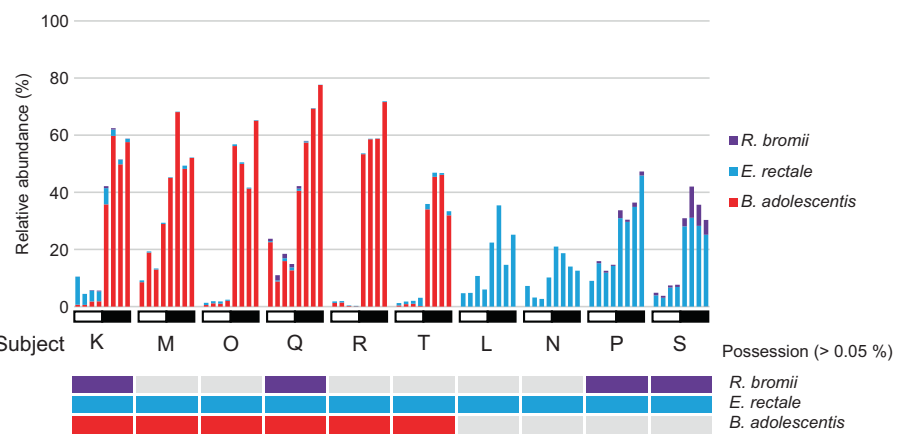

Fig. 2 Two-week intake of potato starch results in expansion of $\boldsymbol{B}$. adolescentis. a Scheme of the pSt intake experiment. b Family-level $16 \mathrm{~S}$ rRNA profiles before and during intake of pSt. Less frequent families (average relative abundance $<1 \%$ ) are shown as others. c Relative frequency of known major starch-utilizing species before and during intake of pSt. The presence ( $>0.05 \%$; colored cells) or absence (gray cells) of the indicated species is shown beneath the graph. Note that $B$. adolescentis was responsible for a large fraction of the increase in Bifidobacteriaceae. d Response of OTUs to pSt. Only OTUs with $>0.01 \%$ average relative abundance are plotted. Those that showed significant differences between the pre-intake and intake periods ( $s$ value $<0.01)$ are labeled by taxon name. The $s$ value represents the "false sign or small" (FSOS) rate, where "small" denotes LogFC between -1 and 1, shown as dashed lines. Values following taxonomic name are \% identity to the reference sequence. Three known starch-utilizing species are shown as colored dots. 
a

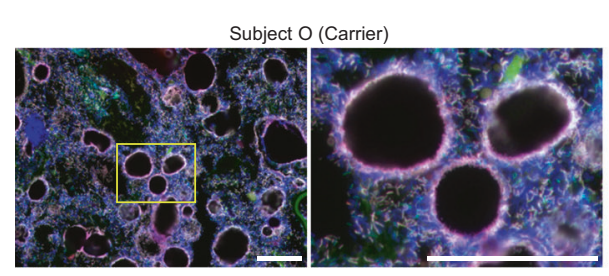

C
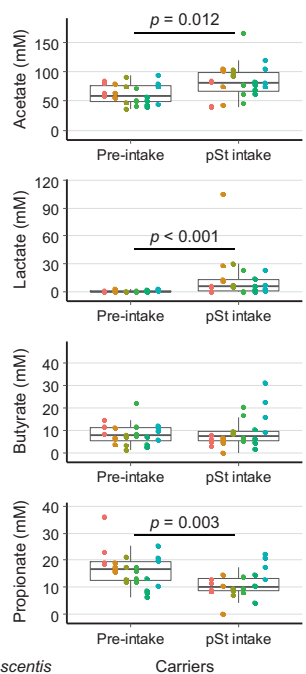

Subject - K M M O Q Q R - T L L N P : S

e

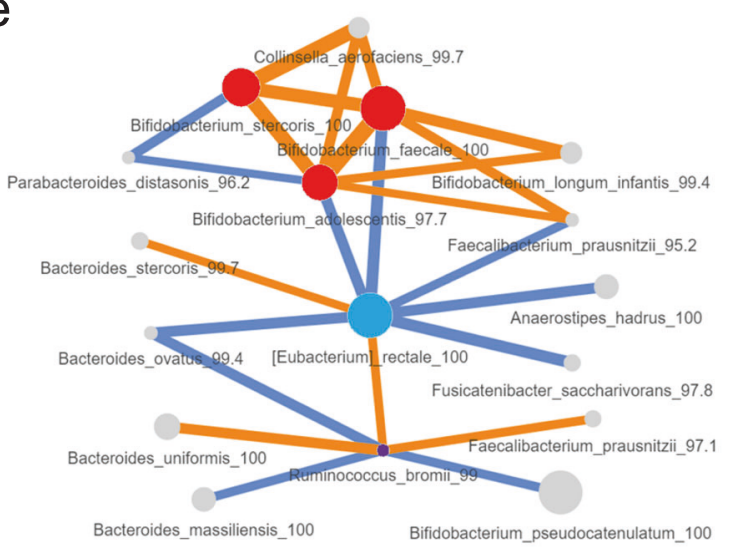

b
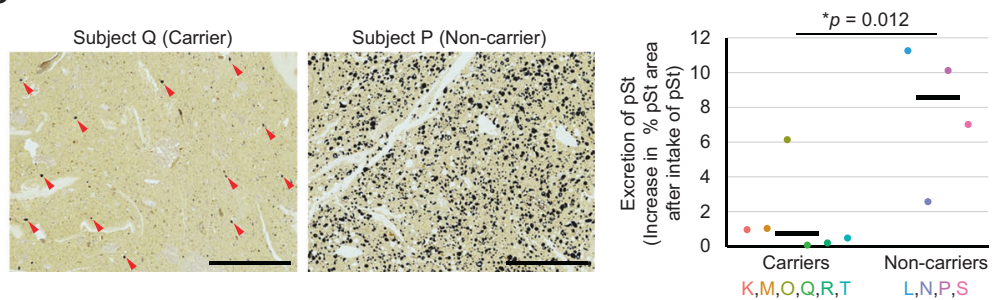

d
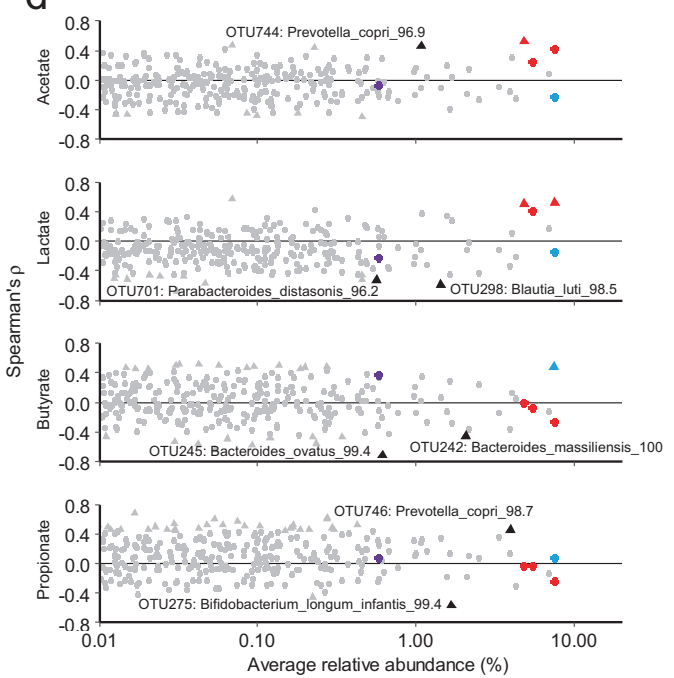
OTUs: $>97 \%$ identity to $B$. adolescentis
OTU: $100 \%$ identity to $R$. bromii $100 \%$ identity to $E$. rectale

A $p<0.05 \quad \bullet \quad p \geq 0.05$

Fig. 3 Metabolic responses of human gut microbiota to two-week intake of potato starch (pSt). a A representative image of colonizing B. adolescentis on fecal starch granules, which are visible as round dark areas (approx. diameter 20-50 $\mu \mathrm{m}$ ). Green: Bifidobacterium, red: B. adolescentis, blue: total bacteria. Bar $=50 \mu \mathrm{m}$. b Excreted starch granules in fecal sections. Representative images of iodine-stained fecal sections during the pSt intake period from a B. adolescentis carrier and non-carrier. Difference in \% area of excreted starch granules between the pre-intake and intake periods is shown in a graph. Horizontal bars indicate medians. * Two-tailed Welch's $t$ test. Arrowheads: starch granules (omitted in non-carrier). Bars $=1 \mathrm{~mm}$. c SCFA concentrations before and during pSt intake in $B$. adolescentis carriers and non-carriers. Elements of box plots are as follows: horizontal bar, median; box limits, upper and lower quartiles; whiskers, $1.5 \times$ interquartile range. $P$ values are based on Wilcoxon test and are adjusted using the Benjamini-Hochberg procedure. $\mathbf{d}$ Taxa that correlate highly with each SCFA as determined by Spearman's correlation analysis. Given that the aim of this analysis was to reveal major producers of each SCFA, only OTUs with $>0.5 \%$ average relative abundance and $p<0.05$ are labeled. Three known starch-utilizing species are shown as colored dots. e Taxa that correlated $(p<0.05)$ with B. adolescentis, E. rectale, or R. bromii as determined by Spearman's correlation analysis. Only OTUs with $>0.5 \%$ average relative abundance and detected in more than half of the samples are shown. Orange: positive correlation, blue: negative correlation. Node size is proportional to the relative abundance of the OTU, and edge width is proportional to the correlation coefficient (Spearman's $\rho$ ).

Surprisingly, though all subjects had $E$. rectale, an increase in this species was observed in all four $B$. adolescentis non-carriers but not in any of the six carriers (Fig. 2c). Similarly, increases in $R$. bromii and $R$. torques were evident only in $B$. adolescentis non-carriers (Fig. 2c and Supplementary Fig. 4). However, since $B$. adolescentis and $E$. rectale were both highly abundant, their increase might lead to compositional problem: non-differential features might appear to be reduced due to the constant-sum 
a

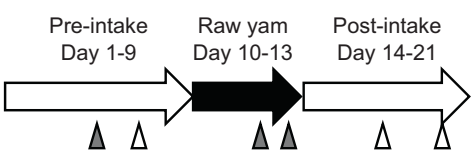

$\triangle$ 16S rRNA profiling + fecal section

$\triangle 16 \mathrm{~S}$ rRNA profiling b

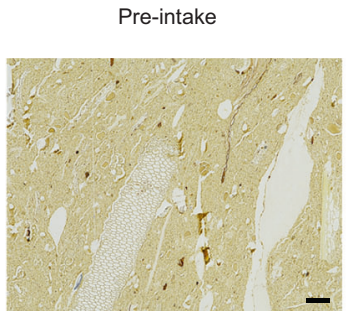

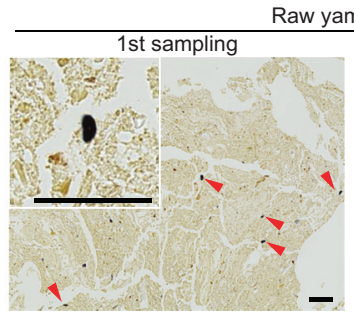

Raw yam intake

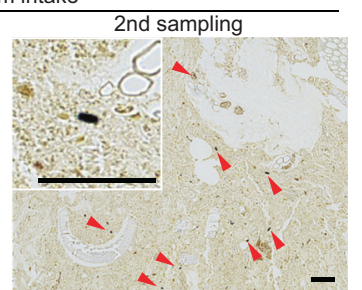

C

Subject G

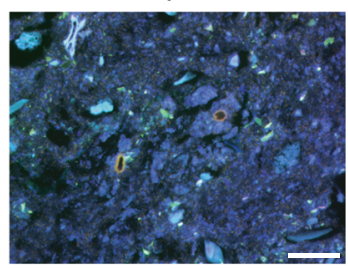

Subject E
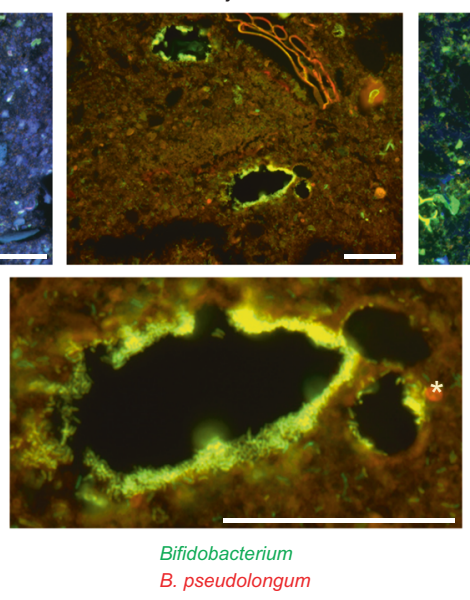

B. pseudolongum

B. pseudolongum
Subject $\mathrm{H}$
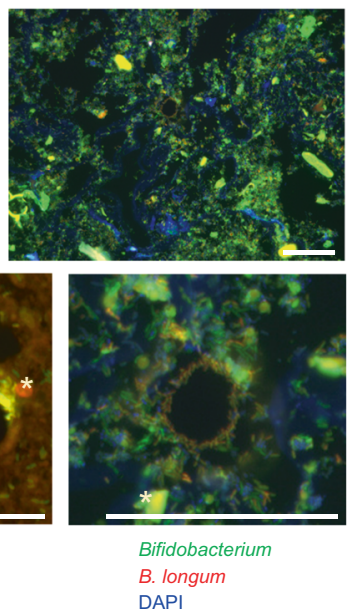

Fig. 4 Colonization of bifidobacterial species on starch granules in feces. a Design of the raw yam intake experiment (ten subjects). b Starch granules were specifically detected during the raw yam intake period. Typical images of iodine-stained fecal sections are shown (specimens from subject B). Bars $=100 \mu \mathrm{m}$. Arrowheads: starch granules. c Colonization of bifidobacterial species on starch granules during the raw yam intake period. Bars $=50 \mu \mathrm{m}$. ${ }^{*}$, Autofluorescence.

constraint in relative abundance. To reduce these effects, we normalized the data by geometric mean of pairwise ratios (GMPR) method, and successfully confirmed that the same conclusion as above was obtained (Fig. 2d). Between B. adolescentis carriers and non-carriers, we found significant differences in gut microbiota metabolism. The amount of undigested pSt granules in fecal sections (Supplementary Fig. 5) was smaller in B. adolescentis carriers than in non-carriers (Fig. 3b). Furthermore, in $B$. adolescentis carriers but not in non-carriers, fecal concentrations of major bifidobacterial metabolites acetic and lactic acids increased and those of propionate decreased upon intake of pSt (Fig. 3c). Instead, a major metabolite of E. rectale, butyric acid, increased in B. adolescentis non-carriers but not in carriers (Fig. 3C). In Spearman's correlation analysis, $B$. adolescentis was the most abundant among operational taxonomic units (OTUs) that were significantly correlated with the concentrations of acetic and lactic acids, and E. rectale was the most abundant among OTUs that were significantly correlated with the concentration of butyrate, suggesting that they produced a major fraction of each SCFA from pSt (Fig. 3d). In another Spearman's correlation analysis, E. rectale was positively correlated with $R$. bromii and negatively with $B$. adolescentis and several butyrate-producing bacteria (Fig. 3e). For $B$. adolescentis, positive correlation was found with $B$. longum and Collinsella aerofaciens. Overall, these results strongly suggest that (i) among all bacterial species detected in this study, $B$. adolescentis most efficiently consumes intestinal pSt granules, which is facilitated by their colonization, to increase its abundance and generation of acetic and lactic acids, and that (ii) E. rectale can increase its abundance upon pSt intake, generating butyrate, but only in the absence of the dominant consumer $B$. adolescentis.

To better understand the cause of the hierarchy between $B$. adolescentis and other species, especially E. rectale, we analyzed fecal sections again to distinguish colonizing and non-colonizing species (Experiment III). For this purpose, we employed another cohort, who consumed lower amounts of starch granules, to identify colonization more easily (Fig. 4a, b). This experiment confirmed that colonization of $B$. adolescentis on starch granules was outstanding among major bifidobacterial species (Table 1, Fig. 4c, Supplementary Fig. 6 and Supplementary note 1$)$. Colonization by $B$. adolescentis was detected in every carrier of the species, and half or more of starch granules were densely colonized by the species. However, unfortunately we could not adequately determine the localization of E. rectale by rRNA-targeted FISH. In most of the specimens analyzed, the signal from $E$. rectal was too weak to identify its location. Presumably, the rRNA of this anaerobic species had already been degraded before fixation, when it was present in the rectum, or after the cells were damaged by exposure to oxygen at the moment of sampling.

Therefore, instead, we checked the distribution of abilities to colonize and utilize pSt granules in major gut bacterial species by isolating them from two subjects who participated in Experiment III. A variety of dominant species including bifidobacteria were isolated, and pSt granule-binding ability and soluble starch-degrading ability were evaluated for at least one strain per species and subject (Supplementary Table 2). Strains with any positive results were included in further analysis of their ability to utilize pSt. Bifidobacterium adolescentis was able to bind to and utilize pSt granules (Table 2), as were most of other strains of the species from different origins (Supplementary Table 3 and Supplementary note 2). Among evaluated isolates other than $B$. adolescentis, the ability to utilize soluble (boiled) pSt was shared by many taxa; all evaluated Bacteroidaceae species and E. rectale. However, pSt granule-binding ability was shared only by E. rectale 
Table 1. Determination of starch granule-colonizing bifidobacterial species.

Colonization on starch granule + positive -- negative (ND: not determined) relative abundance $<0.5 \%$

\begin{tabular}{|c|c|c|c|c|c|c|c|c|c|c|}
\hline Subject ID & B & C & D & G & E & $\mathbf{H}$ & A & $\mathbf{F}$ & 1 & $\mathbf{J}$ \\
\hline Bifidobacterium & + & + & + & + & + & + & - & - & - & - \\
\hline B. adolescentis & $+^{*}$ & $t^{*}$ & $t^{*}$ & $t^{*}$ & & & & & & \\
\hline B. longum & - & - & & - & - & $+^{*}$ & ND & ND & ND & ND \\
\hline B. catenulatum gr. & & - & - & & - & & & ND & & ND \\
\hline B. pseudolongum & & & & & $t^{*}$ & & & & & \\
\hline $\begin{array}{l}\text { Frequency of starch granules } \\
\text { colonized by indicated }\left({ }^{*}\right) \text { taxa }\end{array}$ & high & high & medium & medium & high & low & ND & ND & ND & ND \\
\hline
\end{tabular}

Major species with average relative abundance $>0.5 \%$ were analyzed. Colonization of the indicated taxa was examined using either or both of raw yam intake period samples. If no colonization was detected in a subject using Bifidobacterium-specific probe, species-level FISH for the subject was omitted. Bottom row: frequency of starch granules colonized by the indicated taxa among all granules; high: $>65 \%$, medium: $35-65 \%$, low: $<35 \%$.

Table 2. Binding to and utilization of starch granules by major gut bacterial species.

\begin{tabular}{|c|c|c|c|c|c|c|c|c|}
\hline Family & $\begin{array}{l}\text { Identification } \\
\text { (sequence identity, if < } 97 \% \text { ) }\end{array}$ & Strain & pH control & $\begin{array}{l}\text { Delta pH } \\
\text { Glc or Frc }\end{array}$ & & $\begin{array}{l}\text { Delta pH } \\
\text { boiled potato } \\
\text { starch }\end{array}$ & $\begin{array}{l}\text { delta } \mathrm{pH} \\
\text { raw potato } \\
\text { starch }\end{array}$ & $\begin{array}{l}\text { Binding to } \\
\text { raw potato } \\
\text { starch }\end{array}$ \\
\hline \multirow{4}{*}{ Bifidobacteriaceae } & Bifidobacterium adolescentis & G202 & 6.3 & & -1.9 & -1.9 & -1.7 & ++ \\
\hline & Bifidobacterium catenulatum gr. & F01 & 6.4 & & -2.1 & 0.1 & 0.1 & - \\
\hline & Bifidobacterium catenulatum gr. & G43 & 6.5 & & -2.1 & -0.1 & -0.1 & - \\
\hline & Bifidobacterium longum & F03 & 6.5 & & -2.2 & -0.3 & -0.1 & - \\
\hline Coriobacteriaceae & Collinsella aerofaciens & $\mathrm{F} 47$ & 6.8 & & -2.2 & 0.0 & 0.0 & + \\
\hline \multirow{8}{*}{ Bacteroidaceae } & Bacteroides faecis & G51 & 6.0 & & -1.2 & -1.1 & 0.0 & - \\
\hline & Bacteroides stercoris & G60 & 6.3 & & -1.4 & -1.2 & 0.0 & - \\
\hline & Bacteroides thetaiotaomicron & $\mathrm{F} 57$ & 5.9 & & -1.1 & -0.9 & 0.0 & - \\
\hline & Bacteroides uniformis & F65 & 6.4 & & -1.4 & -1.4 & 0.0 & - \\
\hline & Bacteroides uniformis & G58 & 6.5 & & -1.5 & -0.9 & 0.0 & - \\
\hline & Bacteroides vulgatus & F61 & 6.5 & & -1.6 & -1.5 & 0.0 & - \\
\hline & Bacteroides vulgatus & G52 & 6.6 & & -1.7 & -1.6 & 0.0 & - \\
\hline & Bacteroides xylanisolvens & F59 & 6.2 & & -1.4 & -1.4 & 0.1 & - \\
\hline \multirow{7}{*}{ Lachnospiraceae } & Eubacterium hallii (95.9\%) & F84 & 6.6 & & -1.3 & 0.4 & 0.0 & - \\
\hline & Eubacterium hallii & G74 & 6.8 & & -1.4 & 0.2 & -0.1 & - \\
\hline & Eubacterium rectale & F82 & 6.5 & & $\underline{-0.4}$ & -1.3 & 0.0 & - \\
\hline & Eubacterium rectale & G76 & 6.7 & & $\underline{-0.3}$ & -1.5 & 0.0 & + \\
\hline & Blautia luti (97.0\%) & F22 & 6.2 & & -1.6 & -0.1 & 0.0 & - \\
\hline & Eubacterium ventriosum & F30 & 6.5 & & -1.8 & -0.1 & 0.0 & - \\
\hline & Dorea formicigenerans & G47 & 6.7 & & -1.8 & 0.0 & 0.0 & - \\
\hline Erysipelotrichaceae & Dielma fastidiosa & F60 & 6.8 & & $\underline{-0.9}$ & 0.0 & 0.0 & - \\
\hline \multirow[t]{2}{*}{ (No inocula) } & & & 7.0 & -0.2 & -0.1 & 0.0 & 0.0 & \\
\hline & & & & Delta & & 2.5 & 2.5 & \\
\hline
\end{tabular}

Values indicates $\mathrm{pH}$ after 3-day incubation for culture with no carbon source (control), and the difference in pH between control and corresponding culture with $1 \%$ carbon source. As a positive control, either glucose (Glc) or fructose (Frc) was used. ++ strongly positive, + positive, - negative.

and C. aerofaciens, and it was strain-dependent (Table 2 and Supplementary Table 2). Surprisingly, none of the examined species other than $B$. adolescentis, including $E$. rectale, were able to degrade pSt granules. These results suggest that, among major gut bacterial species, both $B$. adolescentis and $E$. rectale are rare starch granule-binding species, and that the ability to efficiently utilize starch granules is nearly unique to $B$. adolescentis, whereas soluble starch can be utilized by many taxa including Bacteroides.

Finally, since we were unable to obtain any isolates of the known RS2-degrading species R. bromii [30] in the above experiment, we compared the ability of commercially available $R$. bromii strains and the $B$. adolescentis strain obtained in this study to dominate the surface of $\mathrm{pSt}$ in vitro. When an R. bromii strain and a smaller 
a

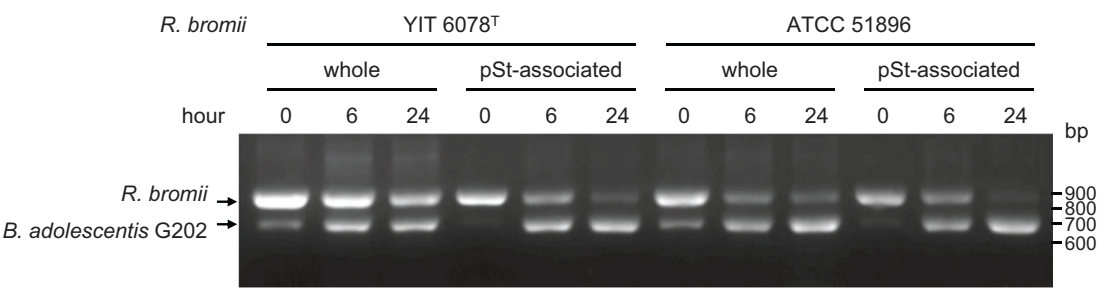

b

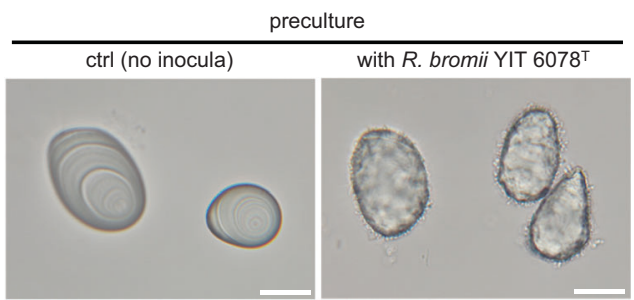

C

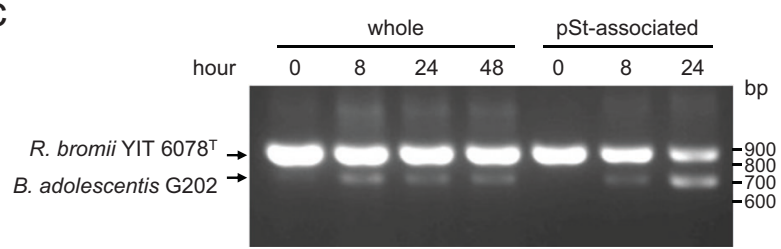

Fig. 5 In vitro competition between B. adolescentis $\mathbf{G 2 0 2}$ and $R$. bromii strains. a $R$. bromii YIT $6078^{\top}$ or $R$. bromii ATCC 51896 and $B$. adolescentis $\mathrm{G} 202$ were simultaneously inoculated into pSt-GAM broth and incubated for the indicated time. Relative abundance of two strains was determined by competitive PCR. b, c R. bromii YIT 6078 ${ }^{\top}$ was grown on pSt-GAM broth for one day and subsequently B. adolescentis G202 was inoculated and the cultures were incubated for the indicated time. $\mathbf{b}$ Bright-field images of pSt without bacteria and $R$. bromii-associated pSt just before inoculation of G202. Bars $=20 \mu \mathrm{m}$. c Relative abundance of the two strains was determined as in a. pSt was completely degraded and no pSt-associated fraction was obtained at $48 \mathrm{~h}$.

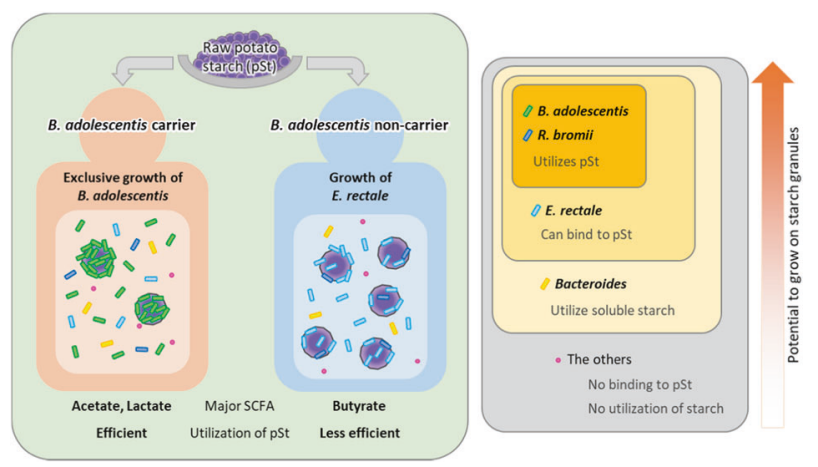

Fig. 6 Proposed model for responses of human gut bacterial species to starch granules. Left: Two consequences of the gut microbiota after ingestion of pSt, which are determined by the posession of $B$. adolescentis. Right: Interspecies hierarchy of potential to grow efficiently using starch granules.

amount of B. adolescentis G202 were inoculated together and incubated, the amounts of both strains in the whole culture became comparable, while G202 accounted for the majority of the population attached to pSt after $24 \mathrm{~h}$ of incubation (Fig. 5a). Even when B. adolescentis $\mathrm{G} 202$ was inoculated after R. bromii YIT $6078^{\top}$ had densely covered the granular surface after one-day culture, the pSt-associated population after coculture contained a large amount of $B$. adolescentis G202, while $R$. bromii continued to constitute the majority of the whole culture (Fig. 5b, c). These results suggest that $B$. adolescentis is able to take over the surface of starch granules in the intestine, even in the presence of the known RS2-degrader R. bromii.

\section{DISCUSSION}

Bacterial strategies and processes for nutrient acquisition have not been surveyed by focusing on microscopic spatial information, at least for normal human gut bacteria. To uncover bacterial life in our gut, we applied histological methods to human fecal material and showed that a specific human-associated dominant bacterial species colonizes starch granules in vivo and effectively responds to their intake by the host. Our results suggest that activity of this species restrained other species with a potential to respond, and thereby determines the major SCFA generated in the gut.

We searched for the locations of major gut bacteria and found prominent and frequent colonization of $B$. adolescentis on starch granules in the human intestinal environment. Although adhesion of $B$. adolescentis onto starch granules has been demonstrated in vitro [31,32] and ex vivo [33], we revealed selective, dense and frequent colonization in the normal human intestinal environment. This colonization was present in every subject who had taken pSt or raw yam starch granules and carried $B$. adolescentis, suggesting that the relationship between starch granules and this species is robust and important for adaptation of this species to human gut.

We found a remarkable increase in $B$. adolescentis abundance after pSt intake in each carrier of the species; the second largest response was found in the butyrate-producing bacterium $E$. rectale. Surprisingly, the response of $E$. rectale to $\mathrm{pSt}$ was observed in all $B$. adolescentis non-carriers but not in carriers, and a similar response was also observed in $R$. bromii. This relationship was consistently detected using GMPR method for normalization, which reduces the compositional problem. Given that the total bacterial count was comparable between the pre-intake and intake periods, the increase in the relative abundance of $B$. adolescentis should reflect an increase in its count. Despite the limited number of subjects, the difference between $B$. adolescentis 
carriers and non-carriers was evident (Fig. 6). The results also showed that the amount of pSt residues and the major SCFAs generated from pSt in the feces depended on whether the subject was a carrier or non-carrier of $B$. adolescentis. Consistently, correlation analysis suggested that $B$. adolescentis, the main increased species in the former, and $E$. rectale, that in the latter, contributed to production of acetic and lactic acids and of butyric acid, respectively. The decrease in propionate in carriers could be explained by a decrease in the abundance of the propionateproducing family Bacteroidaceae or Prevotellaceae (Fig. 2b) [34]. Though the responses of $B$. adolescentis and $E$. rectale to intake of starch granules or similar RS2 food have been reported [9, 28, 35], to our knowledge, this hierarchical relationship where $B$. adolescentis "rules" the activities of other less potent responding species has not been demonstrated. This inter-species relationship could help us predict individualized responses of gut microbiome to pSt and possibly to other starches categorized as RS2. Moreover, our results imply that a single species, $B$. adolescentis, can be a critical factor that determines the diversity of the health effects of starch granules among individuals. About half of the population is estimated to have $B$. adolescentis [36], and the effects of RS2 on metabolic health vary among trials and are not always significant [37, 38]. It is expected that ingestion or suppression (e.g., with a specific glycosidase inhibitor or antibody) of this species will unify the heterogeneous responses of the microbiota, SCFA, and possibly host metabolic parameters to starch granules. This approach will contribute to the efficient use of this potent and easy-to-use food material to fulfill individualized needs of a large fraction of the population. Considering that the levels of $B$. adolescentis and lactate in this study were quite high, the dose of starch granules has to be adjusted according to the desired individual effect. For example, using a low dose to maintain mild changes for a long time would be safe and suitable for metabolic effects, whereas using high doses to induce a large amount of acids may be effective for urgent eradication of some enteric pathogenic bacteria. In addition, potential differences between males and females in microbiota responses to starch granules remain to be addressed, since our data were obtained from male subjects only.

It should be considered that the relationship between $B$. adolescentis and $E$. rectale may be geographical region-specific or strain-specific, given that it was not evident in similar studies in the United States $[9,28,35]$. Indeed, a genomic study indicates that $E$. rectale strains can be divided into several geographically stratified types with different carbohydrate metabolism genes [39]. Future mechanistic and population studies will be necessary for understanding this. In addition to clarifying the relationship between $B$. adolescentis and $E$. rectale, our results imply downstream interspecies interactions. Positive correlation between $B$. adolescentis and $B$. longum suggests that the former species created an environment preferred by Bifidobacterium species, which led to the increase in their relative fitness. Positive correlation between $B$. adolescentis and $C$. aerofaciens suggests crossfeeding of maltose. Maltose is a substrate utilized by every strain of the latter species and can be enriched near $B$. adolescentis-colonized pSt. Our in vitro experiment suggests that some strains of $C$. aerofaciens bind to pSt, supporting this model. In $B$. adolescentis carriers, we detected no significant increases in butyrate, E. rectale, or other butyrate-producing bacteria. These bacteria can convert lactate to butyrate, but their activity seems to be suppressed in our experiment by the strongly acidic environment induced by metabolism of $B$. adolescentis [40]. Meanwhile, we detected an increase in a lactate-utilizing species Megasphaera indica [41] in B. adolescentis carriers (Fig. 2d, Correlation between OTUs of $B$. adolescentis and $M$. indica; $\rho=$ $0.57, p<0.01)$. This increase suggests that $M$. indica rather than butyrate producers could benefit from crossfeeding of lactate in a strongly acidic environment.
The advantage of $B$. adolescentis in utilization of starch granule is substantiated by in vitro activities of dominant species isolated from the participants. These results reinforce that $B$. adolescentis is highly adapted to raw granular starches and suggest that monopolization of the starch surface by adhesion promotes the selective response of the species as it limits expansion of other species, which can increase in abundance in the absence of $B$. adolescentis. The model is substantiated by the results of our in vitro competition assay, which suggest that $B$. adolescentis can take over the pSt surface from the known starch granuledegrading species, $R$. bromii. The acidic environment induced by $B$. adolescentis (especially the presence of lactate, pKa 3.86) may also contribute to the advantage of $B$. adolescentis over other species such as $E$. rectale [40]. We confirmed a previous result $[30,42]$ that $E$. rectale cannot utilize raw pSt in vitro, but it did respond to the intake of raw pSt. This may be explained by the activity of host amylase or amylases from other bacteria. Because $E$. rectale responded to $\mathrm{pSt}$ in the absence (or presence below the detection limit) of $R$. bromii (subjects $\mathrm{L}, \mathrm{N}$ ), there should be another mediator with activity similar to that of $R$. bromii.

Taken together, our results provide a good example of competition for a common but specific food ingredient and hierarchical relationship among major bacteria in the normal human intestine. Our findings could provide a basis for understanding similar interspecific relationship. In situ colonization analysis brought us mechanistic understanding of inter-species hierarchy in response to a nutrient and prompted us to propose future plans for a precise control of microbiota. A similar approach may reveal competition among other species for other nutrients and contribute to our understanding and ability to use the microbiota.

\section{REFERENCES}

1. Gentile C, Weir T. The gut microbiota at the intersection of diet and human health. Science. 2018;362:776-80.

2. Kolodziejczyk AA, Zheng D, Elinav E. Diet-microbiota interactions and personalized nutrition. Nat Rev Microbiol. 2019;17:742-53.

3. David LA, Maurice CF, Carmody RN, Gootenberg DB, Button JE, Wolfe BE, et al. Diet rapidly and reproducibly alters the human gut microbiome. Nature. 2014;505:559-63.

4. Vandeputte D, Falony G, Vieira-Silva S, Wang J, Sailer M, Theis S, et al. Prebiotic inulin-type fructans induce specific changes in the human gut microbiota. Gut. 2017;66:1968-74.

5. Azcarate-Peril MA, Ritter AJ, Savaiano D, Monteagudo-Mera A, Anderson C, Magness ST, et al. Impact of short-chain galactooligosaccharides on the gut microbiome of lactose-intolerant individuals. Proc Natl Acad Sci USA. 2017;114: E367-E375.

6. Matsuki T, Yahagi K, Mori $H$, Matsumoto $H$, Hara T, Tajima $S$, et al. A key genetic factor for fucosyllactose utilization affects infant gut microbiota development. Nat Commun. 2016:7:11939.

7. Rakoff-Nahoum S, Foster KR, Comstock LE. The evolution of cooperation within the gut microbiota. Nature. 2016;533:255-9.

8. Patnode ML, Beller ZW, Han ND, Cheng J, Peters SL, Terrapon N, et al. Interspecies competition impacts targeted manipulation of human gut bacteria by fiberderived glycans. Cell. 2019;179:59-73.e13.

9. Venkataraman A, Sieber JR, Schmidt AW, Waldron C, Theis KR, Schmidt TM. Variable responses of human microbiomes to dietary supplementation with resistant starch. Microbiome. 2016;4:33.

10. Swidsinski A, Loening-Baucke V, Verstraelen H, Osowska S, Doerffel Y. Biostructure of fecal microbiota in healthy subjects and patients with chronic idiopathic diarrhea. Gastroenterology. 2008;135:568-79.

11. Nava GM, Friedrichsen HJ, Stappenbeck TS. Spatial organization of intestinal microbiota in the mouse ascending colon. ISME J. 2011;5:627-38.

12. Nagara $Y$, Takada T, Nagata $Y$, Kado S, Kushiro A. Microscale spatial analysis provides evidence for adhesive monopolization of dietary nutrients by specific intestinal bacteria. PLoS One. 2017;12:e0175497.

13. Takada T, Chinda D, Mikami T, Shimizu K, Oana K, Hayamizu S, et al. Dynamic analysis of human small intestinal microbiota after an ingestion of fermented milk by small-intestinal fluid perfusion using an endoscopic retrograde bowel insertion technique. Gut Microbes. 2020;11:1662-76.

14. Matsuki T, Watanabe K, Fujimoto J, Miyamoto $Y$, Takada T, Matsumoto K, et al. Development of $16 \mathrm{~S}$ rRNA-gene-targeted group-specific primers for the 
detection and identification of predominant bacteria in human feces. Appl Environ Microbiol. 2002;68:5445-51.

15. Bolyen E, Rideout JR, Dillon MR, Bokulich NA, Abnet CC, Al-Ghalith GA, et al. Reproducible, interactive, scalable and extensible microbiome data science using QIIME 2. Nat Biotechnol. 2019;37:852-7.

16. Callahan BJ, McMurdie PJ, Rosen MJ, Han AW, Johnson AJ, Holmes SP. DADA2: High-resolution sample inference from Illumina amplicon data. Nat Methods. 2016;13:581-3.

17. Bokulich NA, Kaehler BD, Rideout JR, Dillon M, Bolyen E, Knight R, et al. Optimizing taxonomic classification of marker-gene amplicon sequences with QIIME 2's q2-feature-classifier plugin. Microbiome. 2018;6:90.

18. Rognes T, Flouri T, Nichols B, Quince $C$, Mahé F. VSEARCH: a versatile open source tool for metagenomics. PeerJ. 2016;4:e2584.

19. Wright ES. Using DECIPHER v2.0 to Analyze Big Biological Sequence Data in R. The R Journal. 2016;8:352-9.

20. Love MI, Huber W, Anders S. Moderated estimation of fold change and dispersion for RNA-seq data with DESeq2. Genome Biol. 2014;15:550.

21. Chen L, Reeve J, Zhang L, Huang S, Wang X, Chen J. GMPR: a robust normalization method for zero-inflated count data with application to microbiome sequencing data. PeerJ. 2018;6:e4600.

22. Zhu A, Ibrahim JG, Love MI. Heavy-tailed prior distributions for sequence count data: removing the noise and preserving large differences. Bioinformatics. 2019;35:2084-92.

23. Hornik K, Hothorn T. exactRankTests: exact distributions for rank and permutation tests. R package version 0.8-31. 2019. Available from: https://cran.r-project.org/ web/packages/exactRankTests/.

24. Benjamini $Y$, Hochberg $Y$. Controlling the false discovery rate: a practical and powerful approach to multiple testing. J R Stat Soc B. 1995;57:289-300.

25. Harrell FE. Hmisc: Harrell miscellaneous. R package version 4.4-2. 2020. Available from: https://cran.r-project.org/web/packages/Hmisc/.

26. Almende BV. visNetwork: Network Visualization using 'vis.js' Library. R package version 2.1.0. 2021. Available from: https://cran.r-project.org/web/packages/visNetwork/.

27. Duranti S, Turroni F, Lugli GA, Milani C, Viappiani A, Mangifesta M, et al. Genomic characterization and transcriptional studies of the starch-utilizing strain Bifidobacterium adolescentis 22L. Appl Environ Microbiol. 2014;80:6080-90.

28. Baxter NT, Schmidt AW, Venkataraman A, Kim KS, Waldron C, Schmidt TM. Dynamics of human gut microbiota and short-chain fatty acids in response to dietary interventions with three fermentable fibers. mBio. 2019;10:e02566-18.

29. Cockburn DW, Orlovsky NI, Foley MH, Kwiatkowski KJ, Bahr CM, Maynard M, et al Molecular details of a starch utilization pathway in the human gut symbiont Eubacterium rectale. Mol Microbiol. 2015;95:209-30.

30. Ze X, Duncan SH, Louis P, Flint HJ. Ruminococcus bromii is a keystone species for the degradation of resistant starch in the human colon. ISME J. 2012;6:1535-43.

31. Crittenden R, Laitila A, Forssell $P$, Mättö J, Saarela M, Mattila-Sandholm $T$, et al. Adhesion of bifidobacteria to granular starch and its implications in probiotic technologies. Appl Environ Microbiol. 2001;67:3469-75.

32. Jung DH, Kim GY, Kim IY, Seo DH, Nam YD, Kang H, et al. Bifidobacterium adolescentis P2P3, a human gut bacterium having strong non-gelatinized resistant starch-degrading activity. J Microbiol Biotechnol. 2019;29:1904-15.

33. Leitch EC, Walker AW, Duncan SH, Holtrop G, Flint HJ. Selective colonization of insoluble substrates by human faecal bacteria. Environ Microbiol. 2007;9:667-79.

34. Louis P, Flint HJ. Formation of propionate and butyrate by the human colonic microbiota. Environ Microbiol. 2017;19:29-41.

35. Martínez I, Kim J, Duffy PR, Schlegel VL, Walter J. Resistant starches types 2 and 4 have differential effects on the composition of the fecal microbiota in human subjects. PLoS One. 2010;5:e15046.

36. Odamaki T, Kato K, Sugahara H, Hashikura N, Takahashi S, Xiao JZ, et al. Agerelated changes in gut microbiota composition from newborn to centenarian: a cross-sectional study. BMC Microbiol. 2016;16:90.

37. Peterson CM, Beyl RA, Marlatt KL, Martin CK, Aryana KJ, Marco ML, et al. Effect of 12 wk of resistant starch supplementation on cardiometabolic risk factors in adults with prediabetes: a randomized controlled trial. Am J Clin Nutr. 2018;108:492-501.

38. Snelson M, Jong J, Manolas D, Kok S, Louise A, Stern R, et al. Metabolic effects of resistant starch type 2: a systematic literature review and meta-analysis of randomized controlled trials. Nutrients. 2019;11:1833.
39. Karcher N, Pasolli E, Asnicar F, Huang KD, Tett A, Manara S, et al. Analysis of 1321 Eubacterium rectale genomes from metagenomes uncovers complex phylogeographic population structure and subspecies functional adaptations. Genome Biol. 2020;21:138.

40. Wang SP, Rubio LA, Duncan SH, Donachie GE, Holtrop G, Lo G, et al. Pivotal roles for $\mathrm{pH}$, lactate, and lactate-utilizing bacteria in the stability of a human colonic microbial ecosystem. mSystems. 2020;5:e00645-20.

41. Lanjekar VB, Marathe NP, Venkata Ramana V, Shouche YS, Ranade DR. Megasphaera indica sp. nov., an obligate anaerobic bacteria isolated from human faeces. Int J Syst Evol Microbiol. 2014;64:2250-6.

42. Cockburn DW, Suh C, Medina KP, Duvall RM, Wawrzak Z, Henrissat B, et al. Novel carbohydrate binding modules in the surface anchored a-amylase of Eubacterium rectale provide a molecular rationale for the range of starches used by this organism in the human gut. Mol Microbiol. 2018;107:249-64.

\section{ACKNOWLEDGEMENTS}

We would like to thank all the participants for their dedication to this study Dr. Takahiro Matsuki and colleagues, and Dr. Kaihei Oki and Dr. Junji Fujimoto for providing $B$. adolescentis strains (eight infant strains and six Belgian strains, respectively); Mr. Yohei Watanabe for support in microbiome data analysis; Ms. Miyuki Katto for experimental support; and Dr. Hiroshi Makino for reviewing manuscript.

\section{AUTHOR CONTRIBUTIONS}

YN and KO designed and directed the study. YN, TT, MSY, and TO conducted experiments. DF and YN performed bioinformatic analyses. $\mathrm{YN}$ wrote the manuscript with input from all authors. $\mathrm{KO}, \mathrm{DF}$, and TO revised the manuscript.

\section{COMPETING INTERESTS}

The authors declare no competing interests.

\section{ADDITIONAL INFORMATION}

Supplementary information The online version contains supplementary material available at https://doi.org/10.1038/s41396-022-01196-w.

Correspondence and requests for materials should be addressed to Yusuke Nagara.

Reprints and permission information is available at http://www.nature.com/ reprints

Publisher's note Springer Nature remains neutral with regard to jurisdictional claims in published maps and institutional affiliations.

\footnotetext{
(i) Open Access This article is licensed under a Creative Commons Attribution 4.0 International License, which permits use, sharing, adaptation, distribution and reproduction in any medium or format, as long as you give appropriate credit to the original author(s) and the source, provide a link to the Creative Commons license, and indicate if changes were made. The images or other third party material in this article are included in the article's Creative Commons license, unless indicated otherwise in a credit line to the material. If material is not included in the article's Creative Commons license and your intended use is not permitted by statutory regulation or exceeds the permitted use, you will need to obtain permission directly from the copyright holder. To view a copy of this license, visit http://creativecommons. org/licenses/by/4.0/
}

(c) The Author(s) 2022 